Potential transmembrane segments

Start Stop Length $\sim$ Cutoff

$\begin{array}{llll}19 & 19 & 1 & \sim 1.7\end{array}$

$\begin{array}{llll}118 & 125 & 8 & \sim 1.7\end{array}$

$\begin{array}{llll}186 & 199 & 14 & \sim 1.7\end{array}$

$\begin{array}{lllll}360 & 364 & 5 & \sim 1.7\end{array}$

"DAS" TM-segment prediction

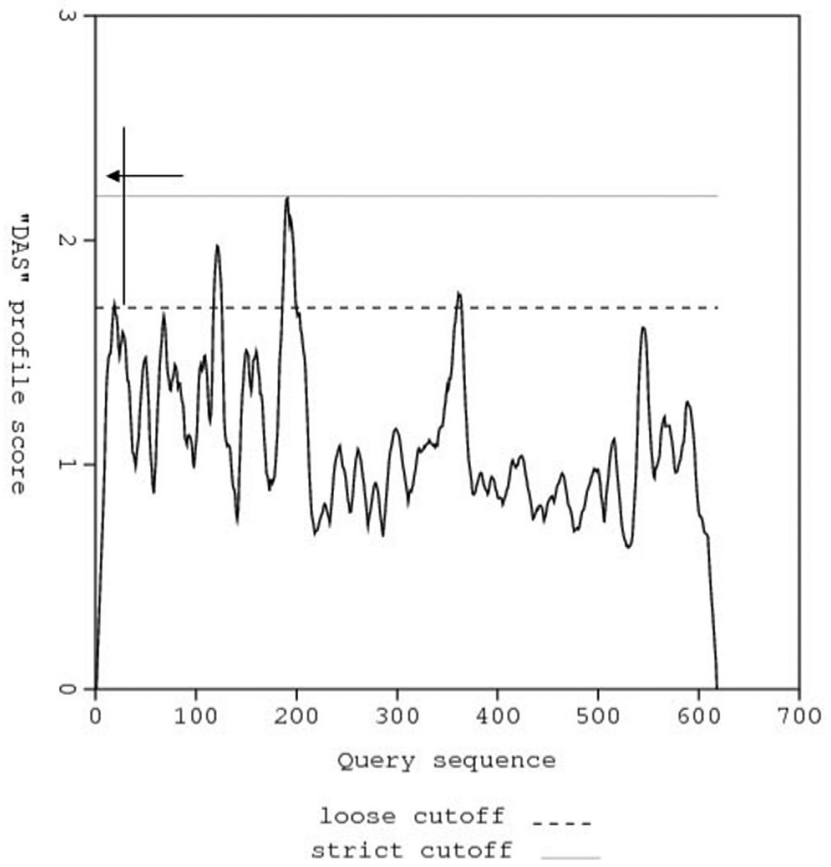

Abstract PS-328 Figure 2 Analysis of transmembrane helices. No transmembrane helice was found in protein PPE16 between the cleavage site (P29) and the N-terminus, as indicated by arrow.

replication evades the innate immune response and becomes latent remains unclear.

Materials and methods RSV persistently infected HEp-2 cells were isolated and the clones were passaged. By using siRNA silence of RIG-I or TRL3, protein levels of SOCS1, SOCS3 and STAT1/2 in the viral persistent cells were checked by western blot, cytokines concentrations in the supernatant of were determined by ELISA, and antiviral genes expression was detected by RT-PCR.

Results The RSV persistent cells always differentiated into two distinct populations characterised by viral permission or resistance respectively. The viral persistent cells produced a low viral titer, resisted wild-type RSV superinfection, and secreted high levels of IFN- $\beta$, Mip- $\alpha$, IL- 8 and Rantes. TLR3, RIG-I and SOCS1 were found to be upregulated. The silence of TLR3 decreased the expression of SOCS1 and the secretion of cytokines.

Conclusion RSV persistent cells are in an inflammatory state that the upregulation of SOCS1 is related to the TLR3 induced signalling pathway, which could be associated with viral persistence.

\section{PS-330 EVALUATION OF DENGUE IGA AND NS1 RAPID TEST AS AN EARLY DIAGNOSTIC TEST FOR DENGUE VIRUS INFECTION}

${ }^{1} \mathrm{C}$ Irianto, ${ }^{2} \mathrm{G}$ Go. ${ }^{1}$ Pediatrics, Mary Chiles General Hospital, Surabaya, Indonesia;

${ }^{2}$ Pediatrics, Mary Chiles General Hospital, Manila, Philippines

10.1136/archdischild-2014-307384.629
Background Dengue is a major health problem. The lack of consensus on excellent rapid diagnostic test for early detection of dengue infection has generated interest to determine the validity of dengue IgA versus NS1 as rapid test in the early diagnosis of dengue with hemagglutination inhibition (HI) as standard reference among paediatric patients up to $<19$ years old seen a private hospital from March, 1, 2012 to October, 30, 2012.

Methods Paired serum samples were examined from 51 patients suspected of dengue with fever of not more than 7 days. There were 29 males and 22 females. Initial blood samples were collected on the first day of consult and tested for $\operatorname{IgA}$, NS1, and HI. Second blood samples for HI were collected 7 days after the initial extraction.

Results From 51 samples, sensitivity of dengue IgA was $80 \%$ with 95\% CI (70-90) while specificity was at 50\% with $95 \% \mathrm{CI}$ (36-64) versus NS1 which showed sensitivity of $27 \%$ with $95 \%$ CI (15-39) and specificity of $67 \%$ with $95 \%$ CI (54-86). IgA rapid test demonstrated $71 \%$ positivity in detecting acute primary dengue infection and $82 \%$ for acute secondary dengue infection. NS1 detected $43 \%$ of primary dengue infection and $24 \%$ of secondary dengue infection.

Conclusions Dengue IgA was more sensitive for early diagnosis of dengue and had better performance in detecting primary and secondary of dengue than NS1.

\section{Preterm Brain Injury - Experimental}

\section{PS-331 SERIAL CRANIAL ULTRASONOGRAPHY OR EARLY MRI FOR DETECTING PRETERM BRAIN INJURY?}

${ }^{1} \mathrm{MMA}$ Raets, ${ }^{1} \mathrm{~A}$ Plaisier, ${ }^{1} \mathrm{GM}$ Ecury-Goossen, ${ }^{1} \mathrm{P}$ Govaert, ${ }^{1} \mathrm{M}$ Feijen-Roon, ${ }^{1} \mathrm{IM}$ Reiss, ${ }^{1}$ LS Smit, ${ }^{2} \mathrm{M}$ Lequin, ${ }^{1} \mathrm{~J}$ Dudink. ${ }^{1}$ Neonatology, Erasmus University Medical Center - Sophia Children's Hospital, Rotterdam, Netherlands; '2Radiology, Erasmus University Medical Center - Sophia Children's Hospital, Rotterdam, Netherlands

\subsection{6/archdischild-2014-307384.630}

Background and aims Magnetic resonance imaging (MRI) is considered imaging method of choice for high-risk preterm infants to assess brain injury. However, MRI scanning in preterm infants is particularly challenging due to safety, logistical and quality issues that limit clinical feasibility. Meanwhile, due to ongoing technical developments and by using additional acoustic windows, advanced serial cranial ultrasonography (CUS) has acquired great clinical value. We hypothesised that dedicated serial CUS is equally effective in diagnosing preterm brain damage as a routine MRI scan at 30 weeks postmenstrual age and excels in clinical feasibility.

Methods We prospectively collected data of 307 infants born $<29$ weeks gestational age. Serial CUS and MRI were performed according to standard clinical protocol. In case of instability, MRI was postponed or cancelled. Brain images were assessed by independent experts and compared between modalities.

Results Serial CUS was performed in all infants, MRI was often postponed $(n=58)$ or cancelled $(n=127)$. Injury was found in 146 infants (47.6\%). Clinical characteristics differed significantly between groups that were subdivided according to timing of MRI. 61 discrepant imaging findings were found. MRI was superior in identifying cerebellar haemorrhages; CUS in detection of acute intraventricular haemorrhage and cerebral sinovenous thrombosis.

Conclusion Advanced serial CUS seems highly effective in diagnosing preterm brain injury, but may miss cerebellar 
abnormalities. Although MRI does identify these lesions, clinical additional value is limited. Improved safety, better availability and tailored procedures are essential for MRI to increase its value in clinical care.

\section{PS-332 BRAIN TISSUE VOLUMES AT TERM-EQUIVALENT AGE IN PRETERM INFANTS: BIOMARKER FOR NEURODEVELOPMENTAL OUTCOME UNTIL 5 YEARS OF AGE}

K Keunen', 'BJM Kooij van, ${ }^{2} \mathrm{P}$ Anbeek, ${ }^{2} \mathrm{I}$ Isgum, ${ }^{1} \mathrm{IC}$ Haastert, ${ }^{1} \mathrm{MA}$ Helvoort, ${ }^{3}$ RAJ Nievelstein, ${ }^{1} \mathrm{~F}$ Groenendaal, ${ }^{2} \mathrm{MA}$ Viergever, ${ }^{1} \mathrm{LS}$ Vries de, ${ }^{1} \mathrm{MJNL}$ Benders, ${ }^{4}$ MJNL Benders. ${ }^{1}$ Neonatology, University Medical Center Utrecht, Utrecht, Netherlands; ${ }^{2}$ Image Sciences Institute, University Medical Center Utrecht, Utrecht, Netherlands; ${ }^{3}$ Radiology, University Medical Center Utrecht, Utrecht, Netherlands; ${ }^{4}$ Centre for the Developing Brain, King's College, London, UK

\subsection{6/archdischild-2014-307384.631}

Objectives To assess the association of brain tissue volumes at term-equivalent age (TEA) with long-term neurodevelopment.

Methods 108 preterm infants (median GA 28.6 weeks; 25.030.9 weeks) were prospectively studied at TEA (median 41.6 weeks PMA; 39.7-43.6). Volumes of eight different tissue types were quantified using an automatic segmentation method (Anbeek, PLOSOne2013)and related to neurodevelopmental outcome using cognitive (CCs), fine motor (FMss), and gross motor scaled scores (GMss) of the BSITD-III at two years corrected age, Griffiths Mental Development Scales (DQ) at age 3.5, and WPPSI at age 5.5. Corrections were made for PMA at scan, intracranial volume and maternal education.

Results Significant results are presented in the table. Both ventricular (Vent) and cortical grey matter volume (CoGM) were inversely related to all included subscales of the BSITD-III and DQ. However, the association at age 3.5 was lost after excluding infants with severe brain lesions (venous infarction, PHVD with neurosurgical intervention, and severe cerebellar haemorrhages). CoGM volume demonstrated a borderline significant inverse correlation with performal IQ at age 5.5 (coefficient-3.2;-6.6-0.08), that did not change after adjustment for severe brain lesions. Cerebellar volume was related to cognitive outcome at 2 and 3.5 years, but the association was mediated by cerebellar injury.

Conclusion Vent and CoGM volumes at TEA may serve as biomarkers for long-term neurodevelopmental outcome in preterm infants. The relationship between larger CoGM volumes and adverse neurodevelopment may reflect disturbances in white matter-CoGM boundaries and warrants further investigation.

\begin{tabular}{lllll}
\multicolumn{6}{l}{ Abstract PS-332 Table 1} \\
\hline & CCs $(n=108)$ & FMss $(n=108)$ & GMss $(n=108)$ & DQ $(n=94)$ \\
\hline Ventricles & $-3.5 ;-6.1-0.9$ & $-0.8 ;-1.3-0.2$ & $-0.5 ;-0.9-0.1$ & $-2.1 ;-3.8-0.4$ \\
Cerebellum & $5.4 ; 1.0-9.7$ & ns & ns & $4.0 ; 1.5-6.5$ \\
CoGM & $-1.6 ;-2.8-0.5$ & $-0.4 ;-0.7-0.2$ & $-0.4 ;-0.5-0.2$ & $-0.9 ;-1.7-0.2$ \\
\hline
\end{tabular}

Numbers reflect coefficients and $95 \%$-confidence intervals

\section{PS-333 DO CLINICAL RISK FACTORS AFFECT WHITE MATTER MICROSTRUCTURAL INTEGRITY (FA) AT TERM EQUIVALENT AGE IN A MULTI-CENTRE COHORT OF PRETERM NEONATES?}

${ }^{1} \mathrm{~J} \mathrm{Schuurmans},{ }^{1} \mathrm{KJ}$ Kersbergen, ${ }^{1} \mathrm{~F}$ Groenendaal, ${ }^{2} \mathrm{~A}$ Leemans, ${ }^{2} \mathrm{MA}$ Viergever, ${ }^{1} \mathrm{~K}$ Keunen, ${ }^{3} \mathrm{~V}$ Fellman, ${ }^{4} \mathrm{AC}$ Berg, ${ }^{1} \mathrm{~F}$ van Bel, ${ }^{1} \mathrm{LS}$ de Vries, ${ }^{5} \mathrm{Mjnl}$ Benders. ${ }^{1}$ Perinatology, Wilhelmina Children's Hospital University Medical Center Utrecht, Utrecht, Netherlands; ${ }^{2}$ Image Sciences Institute, University Medical Center Utrecht, Utrecht, Netherlands; ${ }^{3}$ Pediatrics,
Lund University and Skåne University Hospital Helsinki University, Lund Helsinki, Sweden; ${ }^{4}$ Pediatrics, Lund University and Skåne University Hospital, Lund, Sweden; ${ }^{5}$ Perinatology Centre for the Developing Brain, Wilhelmina Children's Hospital University Medical Center Utrecht King's College London, Utrecht London, Netherlands

\subsection{6/archdischild-2014-307384.632}

Background and aims We aimed to investigate the effect of clinical risk factors on white matter structural integrity at term equivalent age (TEA), as measured by fractional anisotropy (FA). Methods Diffusion tensor imaging of sufficient quality was available for 182 infants (gestational age (GA) $<28$ weeks), scanned at TEA in two centres. FA values of 14 regions (posterior limb of the internal capsule (PLIC), cerebral peduncles, corpus callosum, sagittal stratum, superior/posterior corona radiata, posterior thalamic radiation, left and right side for all) were automatically calculated (ExploreDTI, Leemans ISMRN 2009) using an atlas-based approach (Oishi, NeuroImage 2011). Subjects with overt parenchymal injury were excluded. Clinical characteristics tested against FA in the multivariable linear regression analysis for each region were GA, gender, intra-uterine growth retardation (IUGR), hypotension, mechanical ventilation $>7$ days, morphine (yes/no), post-haemorrhagic ventricular dilatation (PHVD), surgery, postmenstrual age at scanning (PMA) and participating centre. A cut-off value of $p<0.004(0.05 / 14)$ was used to correct for multiple comparison.

Results Statistically significant positive associations with FA were found for PMA in PLIC (right), Superior Corona Radiata and Posterior Corona Radiata (left) and GA in Corpus Callosum, Sagittal Stratum (left), Posterior Thalamic Radiation (left). Statistically significant negative associations with FA were found for Surgery in Sagittal Stratum (right) and IUGR in Posterior Thalamic Radiation (left). Centre was significantly associated with FA in $8 / 14$ brain regions.

Conclusion The microstructure of the preterm brain at TEA depends on GA and PMA at scan, IUGR and surgery. A possible interaction between surgery and morphine warrants further investigation.

\section{PS-334 BIOMARKERS OF DELAYED BRAIN DEVELOPMENT IN A PIG MODEL OF PRETERM BIRTH}

${ }^{1}$ A Bergström, ${ }^{1} \mathrm{~A}$ Rosenørn, ${ }^{1} \mathrm{AD}$ Andersen, ${ }^{1} \mathrm{~T}$ Thymann, ${ }^{1} \mathrm{SS}$ Kaalund, ${ }^{2} \mathrm{E}$ van der Beek, ${ }^{1} \mathrm{P}$ Sangild. 'Clinical and Experimental Nutrition, University of Copenhagen, Frederiksberg C, Denmark; ${ }^{2}$ Early Life Nutrition, Danone Nutricia, Singapore, Singapore

\subsection{6/archdischild-2014-307384.633}

Background and aims Neurodevelopmental seqeulae of premature birth involve cognitive and motor deficits, often persisting into adult life. The molecular mechanisms involved remain yet to be elucidated, but certain regions e.g. cerebellum and neocortex appear particularly sensitive. The current study aimed to evaluate the relevance of Brain derived neurotropic factor (BDNF), involved in the formation of synaptic connexions, and Sonic Hedgehog ( $\mathrm{SHH})$, important for perinatal neuronal differentiation, as potential biomarkers of brain development.

Methods Piglets were born via planned C-section either at full term (gestational age $118 \mathrm{~d}$ ) or 12 days preterm. Euthanization and brain dissection was performed at postnatal day $5(\mathrm{n}=11$, $\mathrm{n}=33)$ and day $26(\mathrm{n}=22, \mathrm{n}=18)$, for terms and preterms respectively. BDNF and $\mathrm{SHH}$ levels were analysed by ELISA in pig cerebellar homogenates. Western blotting (WB) of downstream targets for BDNF (TrkB) and SHH (Patched, Smoothened, Gli-1) were included together with qPCR-array of 84 